SPANISCHE

LITERATURGESCHICHTE 


\title{
SPANISCHE LITERATURGESCHICHTE
}

\author{
unter Mitarbeit von \\ Sebastian Neumeister, Gerhard Poppenberg, \\ Jutta Schütz und Manfred Tietz \\ herausgegeben von Hans-Jörg Neuschäfer
}

Mit 325 Abbildungen

2., erweiterte Auflage 
Die Deutsche Bibliothek - CIP-Einheitsaufnahme

\section{Neuschäfer, Hans-Jörg:}

Spanische Literaturgeschichte / Hans-Jörg Neuschäfer. Unter Mitarb. von Sebastian Neumeister...

- Stuttgart ; Weimar : 2., erw. Aufl.

Metzler, 2001

ISBN 978-3-476-01857-1

ISBN 978-3-476-01857-1

ISBN 978-3-476-02806-8 (eBook)

DOI 10.1007/978-3-476-02806-8

Dieses Werk einschließlich aller seiner Teile ist urheberrechtlich geschützt. Jede Verwertung außerhalb der engen Grenzen des Urheberrechtsgesetzes ist ohne Zustimmung des Verlages unzulässig und strafbar. Das gilt insbesondere für Vervielfältigungen, Übersetzungen, Mikroverfilmungen und die Einspeicherung und Verarbeitung in elektronischen Systemen.

www. metzlerverlag.de info@metzlerverlag.de (C) 2001 Springer-Verlag GmbH Deutschland Ursprünglich erschienen bei J.B. Metzlersche Verlagsbuchhandlung und Carl Ernst Poeschel Verlag GmbH in Stuttgart 2001 


\title{
INHALTSVERZEICHNIS
}

\section{VORWORT VII}

Zur zweiten Auflage $\mathrm{X}$

\author{
MITTELALTER UND SPÄTMITTELALTER \\ (Manfred Tietz)
}

$\mathrm{Zu}$ den Anfängen der spanischen Literatur 1

Der lateinischsprachige Kontext 3

Die Convivencia im "Spanien der drei Kulturen « 5

Die Anfänge der Reconquista 9

Arabische und hebräische Literatur im Andalus 13

Zur Convivencia in der Literatur 16

Die frühen Werke der spanischen Literatur 24

Frühe erzählende Texte in Prosa: zur Rezeption der orientalischen

Weisheitsliteratur 33

Die nicht-fiktionale Prosa: Alfons der Weise, die »Siete Partidas" und die Chroniken 37

Ein spanischer $>$ Ritterroman $:$ El caballero Zifar 39

Die höfische Lyrik im 13. und 14. Jahrhundert 40

Die Auflösung der Convivencia im 14. Jahrhundert 41

Ein Meisterwerk des Hochmittelalters: El libro de buen amor 44

Zeitkritik im späten Schrifttum 46

Die Hauptgattung der spanischen Lyrik: el romance 48

Vom liturgischen und höfischen Fest zu den Frühformen des Theaters 51

Die Aljamiado-Literatur: der Untergang der arabischen Kultur

im Spanien der Christen 54

Zwischen Spätmittelalter und den Konflikten der Moderne 55

Die Lyrik des 15. Jahrhunderts zwischen späthöfischer Tradition und Neuanfang 58

Die Prosadichtung des 15. Jahrhunderts: die novela sentimental 62

Ein Werk der Krise: die Celestina 65

Vom Spätmittelalter zu Humanismus und Renaissance 67

\section{SIGLO DE ORO}

(Gerhard Poppenberg/Sebastian Neumeister/

Hans-Jörg Neuschäfer/Manfred Tietz)

Einleitung (G. Poppenberg) 69

Neue Welt (G. Poppenberg) 82

Religion und Spiritualität (G. Poppenberg) 87

Moralistik (G. Poppenberg) 93

Die Lyrik im Goldenen Zeitalter (S. Neumeister) 103 
Cervantes und der Roman des Siglo de Oro (H.-J. Neuschäfer) 123

Das Theater im Siglo de Oro (M. Tietz) 152

\section{DAS 18. JAHRHUNDERT}

(Jutta Schütz)

Das 18. Jahrhundert in Spanien - el siglo de las luces? 185

Die erste Jahrhunderthälfte: Anfänge einer saufgeklärten` Literatur 194

Die zweite Jahrhunderthälfte: Blütezeit der Aufklärung

und Übergang zur Romantik 200

DAS 19. JAHRHUNDERT

(Hans-Jörg Neuschäfer)

Geschichtlicher Überblick 231

Zwischen Absolutismus und Liberalismus: die Literatur der spanischen Romantik 239

Literatur und Presse: Costumbrismus und Ursprung des Romans 259

Realismus und Naturalismus: die Literatur der Restaurationszeit 272

Vom Krausismus zur Generation von 98: die Auseinandersetzung

über die Erneuerung Spaniens 305

DAS 20. JAHRHUNDERT

(Hans-Jörg Neuschäfer)

Geschichtlicher Überblick 315

Modernismo und 98. Die Abkehr vom Traditionalismus 322

Die 20er und 30er Jahre 337

Exil und Zensur: die Literatur vor und in der Francodiktatur 362

Nach 1975. Tendenzen der spanischen Gegenwartsliteratur 389

BIBLIOGRAPHIE 424

REGISTER 435

BILDQUELLEN 446 


\section{VORWORT}

1932 schrieb Juan Ramón Jiménez, den die Lyriker der Generation von 1927 als ihren Mentor verehrten, für Gerardo Diegos berühmte Anthologie eine Selbstcharakteristik, die in dem schönen Satz gipfelte: "Yo tengo escondida en mi casa, por su gusto y el mío, a la Poesía. Y nuestra relación es la de los apasionados. " ("Ich halte in meiner Wohnung - weil sie es wollte und ich auch - die Poesie versteckt. Und unsere Beziehung ist die der Leidenschaftlichen. «)

Wenn der Literaturgeschichtsschreiber sein Verhältnis zur spanischen Literatur ganz ähnlich empfindet, mag das auf den ersten Blick erstaunen, sind doch literaturwissenschaftliche >Diskurse zwungen noch hingebungsvoll. Die vorliegende Literaturgeschichte ist aber tatsächlich aus doppelter Zuneigung entstanden: zu Spanien und seiner Kultur; und zu den Studentinnen und Studenten, denen ich eben diese Kultur - vielleicht nicht immer por su gusto, aber stets por el mío - zu vermitteln habe.

Am liebsten hätte ich die Literaturgeschichte allein geschrieben. Sie benötigt ja nicht nur Sachverstand; sie braucht auch erzählerische und gestalterische Qualitäten, wenn sie mehr sein will als eine Bestandsaufnahme oder ein lexikonähnliches Kompendium. In den modernen Buchbindersynthesen indes, wo oft mehr als ein Dutzend von Autoren nur für ein kleines Teilstück zuständig sind, kann es zwar zu einer Ansammlung von Fachwissen, aber kaum zu einer zusammenhängenden Geschichte kommen. Nun ist es mit der "zusammenhängenden Geschichte " gewiß selbst so eine Geschichte. Einerseits bietet ein Erzähler noch keine Garantie für eine anschauliche und lebendige Darstellung. Andererseits kann der Geschichtsschreiber, der über den Fortgang oder gar Ausgang der Historia (mit großem $\mathrm{H}$ ) ebensowenig weiß wie jeder andere Mensch, seine historia (mit kleinem h) nur unter Vorbehalt und mit offenem Ende erzählen, ganz abgesehen davon, daß seine Darstellung nicht unvoreingenommen, weil durch persönliche Vorlieben und Phobien mitbedingt und also subjektiv gefärbt und deshalb anfechtbar ist. Es fragt sich aber, ob eine Vielzahl von Autoren größere Objektivität garantiert. Ich glaube es nicht. Denn aus der Vielfalt von partiellen Ansätzen resultiert noch keine höhere Einsicht, sondern bestenfalls eine Pluralität von Ansichten, die nur schwer miteinander $\mathrm{zu}$ verbinden sind und deshalb meist unvermittelt nebeneinander stehen bleiben. Andererseits ist auch dem Einzelnen die Bemühung um Objektivität keineswegs unmöglich. Dennoch: man kann eine Literaturgeschichte nicht wie einen Roman erzählen. Vorrangig bleibt deshalb die philologische Kompetenz. Und da es vermessen wäre zu glauben, man besäße sie in allen Belangen gleich, suchte ich nach einem dritten Weg, der es erlauben würde, die Verantwortung partnerschaftlich aufzuteilen, ohne das Ganze aus den Augen zu verlieren. In Jutta Schütz und Manfred Tietz fand ich Gleichgesinnte. Zu dritt wollten wir dafür sorgen, daß die Spanische Literaturgeschichte bei Metzler in absehbarer Zeit fertig wird und daß gleichwohl größere Teile von ihr zusammenhängend erzählt werden.

Ein Erzäbler oder mebrere 
Aufteilung der Zuständigkeiten

Leitprinzipien der Darstellung
Manfred Tietz sollte für das Mittelalter und den größten Teil des Siglo de Oro; Jutta Schütz fürs 18. Jahrhundert; Hans-Jörg Neuschäfer für das 19. und 20. Jahrhundert, sowie für den Roman des Goldenen Zeitalters zuständig sein. Dabei wurde von vornherein festgelegt, daß die moderne Literatur Spaniens, die in Deutschland am wenigsten bekannt ist, den Schwerpunkt des Bandes bilden sollte. Wir waren uns auch darüber einig, daß man sich auf die kastilischsprachige Literatur zu beschränken hatte. Nicht nur weil schon für sie allein ein Vierhundertseiten-Band kaum ausreicht, sondern auch, weil man die galizische, vor allem aber die katalanische Literatur nicht als Appendix der kastilischen behandeln kann. Ganz abgesehen davon, daß uns für die baskische Literatur, die auch nicht unberücksichtigt bleiben dürfte, die allernotwendigste Voraussetzung fehlt: die Kenntnis der Spache.

Im wesentlichen ist dieser Plan realisiert worden; wegen Arbeitsüberlastung von Manfred Tietz und mit Rücksicht auf die eingegangene Terminverpflichtung haben wir erst in letzter Minute noch die Hilfe von Sebastian Neumeister und Gerhard Poppenberg in Anspruch genommen, denen ich besonders dankbar dafür bin, daß sie die schwierige Aufgabe übernahmen, Teile des Siglo de Oro ohne die dafür eigentlich notwendige Muße zu bearbeiten.

Ein Wort zu den Vorsätzen, die uns bei unserer gemeinsamen Arbeit geleitet haben (ob sie verwirklicht wurden, wird sich, wie bei allen guten Vorsätzen, erst hinterher und im Urteil der Benutzer herausstellen): Oberstes Prinzip war, verständlich, übersichtlich und möglichst so zu schreiben, daß der Band nicht nur zum Nachschlagen, sondern auch zum Hin- und Herblättern und endlich zur Lektüre größerer Abschnitte einlädt, vor allem aber, daß er den Leser dazu anregt, sich mit der Literatur, von der in ihm die Rede ist, direkt zu beschäftigen. Es sollte außerdem möglich sein, sich sowohl über Sachthemen - zum Beispiel »Was ist die Novela Picaresca?« oder "Welche kulturellen Folgen hatte die Islamisierung der Iberischen Halbinsel? " - als auch über wichtige Einzeltexte - den »Libro de buen amor etwa, den "Don Quijote«, den "Don Juan « oder die »Regenta detailliert und im Zusammenhang zu unterrichten. Ferner wird angestrebt, daß der Leser sich ein Bild von der spanischen Literatur bis in die unmittelbare Gegenwart hinein machen kann, wobei auch die neuen Medien - im 18. und 19. Jahrhundert die Kolportageliteratur und das Stundentheater, im 20. das Kino - gebührende Berücksichtigung finden. Das bedeutet auch, daß unsere Darstellung keinem engen Literaturbegriff (der in Spanien ohnehin nie so maßgebend wurde wie in Deutschland) verpflichtet ist, zumal wir die Literatur als "Lebensäußerung " betrachten, die nicht losgelöst von den historischen Umständen zu verstehen ist, aus denen sie hervorgegangen ist und auf die sie zurückwirkt: man kann den »Cid « nicht verstehen, wenn man nichts von der Reconquista weiß; Lope und Calderón nicht ohne den Bedingungsrahmen des spanischen Theaterwesens auf der einen und die theologischen Grundsatzdebatten auf der anderen Seite; die Gegenwartsliteratur nicht ohne die tiefen Spuren, die der Bürgerkrieg hinterlassen hat. Dies alles rechtfertigt es auch, noch immer die Literaturgeschichte eines beschränkten Raumes, hier der spanischsprachigen Iberischen Halbinsel, für sich allein zu betrachten. Das hat nichts mit der Propagierung einer "Nationalliteratur " zu tun, sondern erklärt sich schlicht aus dem Umstand, daß geschichtliche Erfahrungen nicht nur zeit- sondern auch orts- und vor allem sprachbedingt sind. Wenngleich zuzugeben ist, daß die Globalisierung von Erfahrungen rasant voranschreitet und in Zukunft sicher auch Litera- 
turgeschichten vor neue Probleme stellt, ist die "spanische Literatur", die (fast) immer erstaunlich weltoffen war und viele internationale Anregungen aufnahm (zugleich aber auch großen Einfluß auf andere Literaturen ausübte), noch nicht an ihr Ende gelangt. Vorderhand lohnt es sich also, ihrer 'Differenzqualität< nachzuspüren und dafür zu sorgen, daß nicht in Vergessenheit gerät, was wir ihr zu verdanken haben.

Spaniens Literatur aus ihren ganz eigenen historischen Voraussetzungen zu erklären und ihre `Andersartigkeit` (im Vergleich zu dem für uns Gewohnten und Selbstverständlichen) spürbar zu machen, heißt aber auch, auf Wesensdeutungen, auf globale Sinnzuweisungen und auf andere Fundamentalismen ebenso zu verzichten wie auf eine allein seligmachende Erklärungsmethode. Die eigentliche Stärke der Literatur liegt ja nicht in der Entfaltung einer abstrakten Idee, sondern viel mehr darin, den jeweiligen Gegebenheiten einen Sinn gleichsam probeweise zu unterstellen und ihnen gegenüber Spielräume zu schaffen, die dem Leser (oder Zuschauer/Hörer) eine Ahnung von Freiheit und Selbstbestimmung vermitteln. $\mathrm{Zu}$ zeigen, welche Spielräume in anderen Kulturen geschaffen und welche Alternativen der Orientierung damit auch für uns selbst zur Verfügung gestellt wurden, gehört gewiß zu den schönsten und notwendigsten Aufgaben der Literaturgeschichtsschreibung.

Zum Abschluß einige Hinweise zum Gebrauch des Bandes. Im PersonenRegister findet man die Namen von Autoren, von denen im Text die Rede ist. Unter die Autorennamen sind die zu ihnen gehörenden Werktitel plaziert, sofern sie im Text der Literaturgeschichte eingehend besprochen werden.

Die eigentliche Darstellung folgt dem Faden der Chronologie und benutzt darüber hinaus traditionelle Einteilungsbegriffe, über deren Zulänglichkeit man sich streiten kann, die aber nicht durch praktischere ersetzt sind. Die Orientierung wird - auch ohne Sachregister, das es in den Metzler-Literaturgeschichten nicht gibt - zudem durch eine dreigeteilte Hierarchie von Überschriften und durch Marginalien am Rand des Textes erleichtert. Auch die Abbildungen sollen das Textverständnis unterstützen. Vor jedem großen Einschnitt (Mittelalter, Siglo de Oro, 18.-20. Jahrhundert) werden die historischen Rahmendaten in Erinnerung gebracht, in denen sich die literaturgeschichtliche Erörterung bewegt. Diese selbst ist nicht auf Vollständigkeit aus (dazu gibt es Literaturlexika wie das von Gullón oder die großen Kompendien wie die von Rico oder Alborg). Wir haben - bei dem beschränkten Raum, der uns zur Verfügung stand - ein exemplarisches Vorgehen bevorzugt, bei dem emblematische Autoren und Texte auf Kosten anderer gründlich, bisweilen sogar ausführlich dargestellt werden. - Die Lebensläufe der Verfasser werden zwar nicht immer erörtert; sie werden aber, sofern sie aufschlußreich sind, keineswegs verschmäht: es soll nicht der Eindruck entstehen, als seien Autoren bloße Medien. Vielmehr kann gerade mit Hilfe der Biographie oft anschaulich gemacht werden, in welcher Weise Texte den Lebensumständen abgewonnen wurden.

Für die Zeitspanne zwischen dem Siglo de Oro und dem 19. Jahrhundert war es relativ einfach, plausible Unterteilungen für den darzustellenden Stoff zu finden: für diese Zeit gibt es einen Konsens über die wichtigsten Autoren, die kanonisierten Texte und die Hierarchie von Gattungen. Für das Mittelalter, das spanische zumal, stellt sich die Frage der Einteilung anders; sie ist auch umstrittener. Wir haben uns hier für den Leitfaden der "Convivencia « dreier Kulturen entschieden. Auch im 20. Jahrhundert ist die Organisation des zu Erzählenden ein Problem. Denn hier wird einerseits
Hinweise für die Benutzung des Bandes 
das traditionelle System der Poetik außer Kraft gesetzt, und andererseits gibt es noch keine Übereinkunft über den Textkanon; möglicherweise wird es ihn auch in Zukunft nicht mehr geben. Deshalb waren auch hier andere Leitideen in den Vordergrund zu stellen, allen voran die für Spanien besonders schmerzliche Abkehr vom Traditionalismus und die schwierige Hinwendung zu einer eigenen Moderne. Überall dort, wo neue Wege der Stofforganisation oder der historischen Einteilung gegangen werden, wird im übrigen im Text selbst eine Begründung dafür gegeben.

Es ist leider anzunehmen, daß - trotz sorgfältiger Überprüfung - auch in dieser Literaturgeschichte Fehler vorkommen; möglicherweise nicht nur Druckfehler. Wir bitten die geneigte Leserschaft um Nachsicht und um sachdienliche Hinweise.

Vielen Personen wäre an dieser Stelle zu danken. Ich beschränke mich stellvertretend auf zwei: Markus Trapp, der in Saarbrücken unermüdlich die Fäden zusammenhielt, und Dr. Oliver Schütze, der ein Beispiel dafür ist, daß es doch noch Verlagslektoren gibt.

\section{Zur zweiten Auflage}

Neben kleineren Korrekturen in allen Teilen und einer überarbeiteten Bibliographie bietet die zweite Auflage der Spanischen Literaturgeschichte eine neu konzipierte und erheblich, nämlich auf über das Doppelte erweiterte Darstellung der spanischen Gegenwartsliteratur, die jetzt bis zum Jahr 2000 und damit weiter reicht als andere Literaturgeschichten. Verschiedene Trends, die sich bei der Vorbereitung für die erste Auflage nur abzuzeichnen begannen - etwa das neue Interesse an der Historie und die von den Marktmechanismen erzwungene Umorientierung aufs große Publikum - haben sich inzwischen konsolidiert und können deshalb jetzt vertieft behandelt werden.

Evelyn Schorr (Saarbrücken) sei für die Neubearbeitung des Registers gedankt; Ursula Jung (Bochum) gilt die Anerkennung aller Autoren für die ebenso spontane wie erschöpfende Auflistung von Druck- und anderen Fehlern, die sich in die erste Auflage eingeschlichen hatten.

Saarbrücken, im März 2001

Hans-Jörg Neuschäfer 